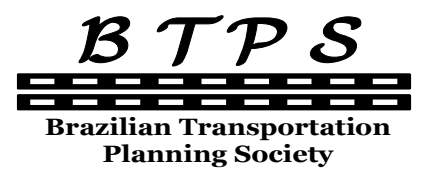

Journal of Transport Literature

Vol. 8, n. 4, pp. 329-349, Oct. 2014

Research Directory
JTL | RELIT

www.journal-of-transport-literature.org ISSN 2238-1031

\title{
Avaliação de processos licitatórios de embarcações de apoio marítimo offshore com utilização de análise envoltória de dados
}

[Evaluation of bidding processes for hiring offshore support vessels using data envelopment analysis models]

\author{
Gustavo da Silva Maciel , Gilson Brito Alves Lima, \\ Lidia Angulo Meza, Silvio Figueiredo Gomes Júnior* \\ Petrobrás - Brazil, Fluminense Federal University - Brazil, \\ Fluminense Federal University - Brazil, State University Center of the West Zone, UEZO - Brazil
}

Submitted 30 Oct 2013; received in revised form 6 Mar 2014; accepted 8 Jun 2014

\begin{abstract}
Resumo
O trabalho apresenta uma proposta de utilização da Análise Envoltória de Dados (DEA) em processos licitatórios para contratação de embarcações tipo PSV (Platform Supply Vessel) de apoio marítimo pela Petrobras. A relevância do trabalho está em considerar as variáveis econômicas e técnicas envolvidas no processo, em alternativa ao processo atual de contratação, que considera apenas o menor preço. A escolha da proposta de menor preço pode gerar custos extras ao longo do tempo, pois não considera aspectos técnicos na avaliação das propostas concorrentes. Utiliza-se o modelo DEA CCR para análise das propostas concorrentes com o uso complementar da fronteira invertida para o cálculo da eficiência. A vantagem desta proposta é que são considerados os aspectos econômicos e técnicos sem qualquer interferência do decisor. Os resultados mostram que a melhor proposta é a embarcação HW, única com eficiência máxima, seguida da embarcação LT. É feita ainda uma comparação dos resultados obtidos pelo modelo DEA proposto com um processo licitatório real.
\end{abstract}

Palavras-Chave: análise envoltória de dados, embarcações, fronteira invertida, petrobras, processos licitatórios.

\begin{abstract}
This paper proposes the use of Data Envelopment Analysis (DEA) models in bidding processes for hiring offshore support vessels type PSV (Platform Supply Vessel) for Petrobras. The relevance of the work lies in taking into account economical and technical variables as an alternative to the current process of hiring, which selects the bid with the lowest price. The choice of the lowest bid can generate extra costs over time, because it does not take into account technical aspects in the evaluation of competing proposals. We use the CCR DEA model to evaluate the competing proposals using also the inverted frontier technique. The advantage of this approach is that technical and economic aspects are taking into account and this is done without any value judgement from the decision maker. The results show that the best bid is the HW vessel (only one with maximum efficiency) then the vessel LT. Also, we compare the results of the DEA proposed approach with a real bidding process.
\end{abstract}

Key words: data envelopment analysis, vessels, inverted frontier, petrobras, bidding processes.

*Email: silviofgj@gmail.com.

\section{Recommended Citation}

Maciel, G. S., Lima, G. B. A., Angulo Meza, L. and Gomes Júnior, S. F. (2014) Avaliação de processos licitatórios de embarcações de apoio marítimo offshore com utilização de análise envoltória de dados. Journal of Transport Literature, vol. 8, n. 4, pp. 329-349.

- JTL/RELIT is a fully electronic, peer-reviewed, open access, international journal focused on emerging transport markets and published by BPTS - Brazilian Transport Planning Society. Website www.journal-of-transport-literature.org. ISSN $2238-1031$. 


\section{Introdução}

A Petrobras, como instituição pertencente à Administração Pública brasileira, segue as normas estabelecidas e utiliza a licitação em diversas atividades. Entre elas, pode-se citar o planejamento logístico para transporte de cargas e suprimentos, indispensável para a continuidade operacional de plataformas de exploração e produção de petróleo e gás natural. Nessa atividade, são utilizadas embarcações de apoio marítimo ao longo de toda a costa brasileira onde se localizam bacias de exploração e produção da Petrobras. As embarcações de apoio marítimo são recursos críticos que têm tido uma demanda crescente no mercado brasileiro de produção de petróleo e gás offshore e, com o desenvolvimento dos campos da camada do Pré-sal, a expectativa é de que, nos próximos anos, esse nicho esteja ainda mais alavancado. Para ressaltar a complexidade da atividade, atualmente para as regiões Sul e Sudeste a Petrobras tem contratadas 115 embarcações, de cerca de 30 fornecedores diferentes, com custo mensal de frota de R\$ 112 milhões, o que representa um gasto contratual num período de dois anos de cerca de $\mathrm{R}$ \$ 2.7 bilhões. Tais valores representam a importância da aplicação de um método adequado aos processos de contratação de embarcações de apoio marítimo da Petrobras.

Assim, o objetivo deste trabalho é avaliar os processos licitatórios da Petrobrás para contratação de embarcações do tipo PSV 3000 TPB, ou seja, que tenham capacidade de carga de 3000 toneladas através a utilização da técnica de Análise Envoltória de Dados (DEA). O uso da técnica se justifica por ser capaz de considerar variáveis econômicas e técnicas envolvidas no processo, além de não ser necessária a intervenção do decisor na atribuição dos pesos destas variáveis. Assim, o mérito deste trabalho é apresentar uma alternativa pouco subjetiva para o critério do menor preço em licitações.

Por permitir a incorporação de variáveis não só econômicas, DEA se apresenta como uma forma alternativa de avaliar propostas em um processo licitatório, que atualmente é realizado apenas considerando a variável preço. Apesar de parecer vantajosa num primeiro momento, a contratação de propostas pelo menos preço pode trazer consequências negativas como, por exemplo, altos custos com manutenção a longo prazo. 
Apesar do método se mostrar apropriado ao problema em estudo, pode-se constatar que existem poucos trabalhos que abordam a seleção de propostas em processos licitatórios utilizando DEA. Alguns exemplos de sua aplicação podem ser vistos nos trabalhos de El-Mashaleh (2013) e (2010), Falagario et al. (2012), Li et al. (2010) e Shuang et al. (2011). No Brasil, uma avaliação prévia destes processos licitatórios pode ser vista no trabalho de Maciel e Angulo Meza (2012). No entanto, no trabalho publicado em 2012, os autores não incluíam duas variáveis que são consideradas importantes quando se consideram aspectos técnicos e de segurança. Assim, este trabalho pode ser considerado como uma evolução da discussão apresentada no trabalho anterior.

Dada a natureza da pesquisa, pode ser considerada como exploratória e se iniciou com um processo de sondagem, a fim de aprimorar ideias, descobrir intuições e, posteriormente, construir hipóteses. Por ser uma pesquisa bastante específica, esta assume a forma de um estudo de caso, sempre em consonância com outras fontes que darão base ao assunto abordado, como é o caso da pesquisa bibliográfica. Os dados quantitativos utilizados no estudo são oriundos de processos licitatórios já ocorridos e divulgados pela Petrobras.

O trabalho está estruturado da seguinte forma: a Seção 1 faz uma revisão da utilização da metodologia DEA proposta em processos licitatórios; a Seção 2 descreve os princípios de DEA, os modelos clássicos e o método da fronteira invertida e a Seção 3 apresenta o estudo de caso, descrevendo a modelagem e a análise e discussão dos resultados.

\section{Análise Envoltória de Dados em Processos Licitatórios}

Na elaboração de licitações, mesmo com objetivos distintos, atualmente contrata-se a proposta que representa o menor valor ofertado, ou seja, que corresponde, a princípio, ao menor valor a ser gasto pelo órgão público, comumente chamado de valor do dinheiro (Ohno e Harada, 2006). Entretanto, existe uma tendência crescente de se permitir mais liberdade na análise da capacidade dos fornecedores (ofertantes do processo licitatório), já que nem sempre a menor oferta pode ser exequível do ponto de vista técnico (Leenders et al., 2006).

Segundo Saranga (2007), durante as duas últimas décadas, DEA estabeleceu-se como uma ferramenta muito útil para estimar a eficiência do desempenho das unidades tomadoras de 
decisão (DMU - Decision Making Units), tanto no setor público quanto no setor privado. Alguns exemplos da aplicação de DEA podem ser vistos nos trabalhos de Dai e Kuosmanen (2014), Menegaki (2013), Li et al. (2013) e Gomes et al. (2009). Mais especificamente, no setor de transporte marítimo tem-se os trabalhos de Caillaux et al. (2011) para a seleção de portos de capotagem de transporte de contêineres no Mercosul, além dos trabalhos de Haralambides (2010), Ferrari e Basta (2009), Managi (2007), Garcia-Alonso e Martin-Bofarull (2007), entre outros.

De acordo com Lorentziadis (2010), a utilização de DEA é adequada na avaliação da competência e do desempenho de fornecedores, podendo classificá-los em eficientes ou ineficientes e, por essa razão, pode ser usado como um primeiro estágio de pré-qualificação para o método de seleção dos licitantes (Narasimhan et al., 2001). Outra característica de DEA e descrita por Zhu et al. (2007) é a flexibilidade e simplicidade da metodologia na definição dos pesos, já que tal etapa não sofre interferência do decisor, já que, em muitos problemas, a definição de pesos é uma tarefa bastante árdua. Uma forma alternativa de resolver a problemática de atribuição de pesos pode ser encontrada no trabalho de Fernandes et al. (2013).

Como descrito em Adler et al. (2002) e Angulo Meza e Estellita Lins (2002), DEA permite estabelecer o ranking das DMUs através dos seus índices de eficiência. Neste contexto, podemos citar os trabalhos de Gabriele et al. (2013), Cortez et al. (2013) e Neto (2013). Assim, como sugerido por Lorentiziadis (2010), os índices de eficiência também poderiam ser utilizados em processos licitatórios para ordenar os licitantes. Apesar dessa sugestão, Lorentziadis (2010) desenvolveu apenas um método multicritério para auxílio dos processos licitatórios, com foco na determinação dos pesos de julgamento após a abertura dos envelopes lacrados.

\section{Fundamentação Teórica}

\subsection{Análise Envoltória de Dados (DEA)}

Originalmente elaborada por Charnes, Cooper e Rhodes (1978), Data Envelopment Analysis (DEA) ou Análise Envoltória de Dados é uma técnica que, a partir de dados observados, utiliza recursos de programação linear para mensurar a eficiência de unidades tomadoras de decisão 
(Decision Making Units - DMUs) que transformam insumos em produtos (Bougnol et al., 2010).

Ao contrário dos chamados métodos paramétricos, a Análise Envoltória de Dados não pressupõe uma relação funcional pré-definida entre os recursos e o que foi produzido (Soares de Mello et al., 2005). Além disso, formalmente, DEA prima por ser uma metodologia de fronteira e, não, de tendência central (Cooper et al., 2000). Portanto, é diferente de métodos paramétricos como a Análise de Regressão porque não desconsidera pontos “outliers", ou seja, que se localizam fora de uma linha de tendência. Ao contrário, em DEA esses pontos "fora da curva" também compõem a medida de eficiência de cada DMU já que considera que o máximo que poderia ter sido produzido foi obtido por meio da observação das unidades mais produtivas (Aizemberg et al., 2011).

Pereira (1995) destaca algumas características da metodologia DEA que reforça sua vocação como uma das principais ferramentas para a análise de eficiência:

- Constrói uma fronteira de eficiência a partir da análise de todas as variáveis em estudo;

- Não necessita da definição a priori de uma função de produção;

- O cálculo da eficiência é feito a partir da consideração de diferentes combinações de inputs e outputs;

- Fornece um conjunto de referência para cada DMU ineficiente.

Embora várias formas da fronteira possam ser determinadas, existem dois modelos que são considerados clássicos: o modelo CCR (também conhecido por CRS ou constant returns to scale) que trabalha com retornos constantes de escala (Charnes et al., 1978) e o modelo BCC, também chamado de VRS (variable returns to scale) que considera situações de eficiência de produção com variação de escala e não assume proporcionalidade entre inputs e outputs, permitindo a introdução do conceito de economia de escala.

O índice de eficiência é calculado em função da forma de projeção das DMUs ineficientes na fronteira, em função da orientação do modelo. É possível orientá-lo de duas formas: orientação a inputs ou orientação a outputs. Se um modelo é orientado a inputs, significa que as DMUs tentarão atingir a fronteira realizando uma diminuição de seus recursos, sem que seus resultados 
sejam alterados. Se um modelo é orientado a outputs, as DMUs tentarão atingir a fronteira maximizando seus resultados, mantendo constantes seus recursos disponíveis.

DEA tem encontrado crescente aplicação por conseguir compor uma única medida (eficiência) a partir de várias variáveis (insumos e produtos) não necessariamente correlacionadas, para comparação relativa das diferentes unidades de produção. Importante salientar que em DEA o termo produção possui sentido amplo e não está necessariamente relacionado a nenhum tipo de produção física (Soares de Mello et al., 2008). Além disso, uma vantagem de DEA é o fato de ela permitir que as variáveis (recursos e produtos) com que as DMUs serão comparadas sejam quaisquer, desde que sejam quantitativas e iguais para todas elas (unidades monetárias, recursos humanos, etc.).

Uma das vantagens dos modelos DEA é permitir fazer ordenações sem depender de opiniões de decisores. Entretanto, estes modelos são extremamente benevolentes com as unidades avaliadas. Assim, as DMUs podem ser eficientes ao considerar apenas algumas das variáveis, aquelas que lhes são mais favoráveis (atribuindo pesos zero às demais variáveis). Essa característica de benevolência dos modelos DEA faz com que ocorram empates para as unidades $100 \%$ eficientes, o que provoca uma baixa discriminação entre as DMUs.

Uma das formas de aumentar a discriminação entre as DMUs nos modelos DEA é a utilização da fronteira invertida, que é uma avaliação pessimista das DMUs. Sua utilização ocorre devido à simplicidade de utilização, diferentemente de outras técnicas conhecidas em DEA como Cross Evaluation (ou avaliação cruzada) (Doyle e Green, 1994; Sexton et al., 1986) e MCDEA (X.B. Li e Reeves, 1999), permitindo contornar a tendência de DEA em atribuir vários pesos nulos. Seu conceito foi introduzido por Yamada et al. (1994) e Entani et al. (2002). Para sua utilização, faz-se uma troca dos inputs com os outputs do modelo original. A fronteira invertida é composta pelas DMUs com as piores práticas gerenciais (podendo ser chamada de fronteira ineficiente). Pode-se igualmente afirmar que as DMUs pertencentes à fronteira invertida têm as melhores práticas sob uma ótica oposta (Lins et al., 2005).

Os tópicos a seguir descrevem com mais detalhes o modelo DEA CCR e a técnica da Fronteira Invertida. 


\subsection{Modelo CCR}

Em 1978, Charnes, Cooper e Rhodes formalizaram o modelo inaugural de DEA: o modelo CCR. É um modelo multidimensional (múltiplos recursos e múltiplos produtos) que constrói uma superfície linear por partes, não paramétrica, envolvendo os dados (Soares de Mello et al., 2005). Em sua formulação matemática considera-se que cada DMU $k$ é uma unidade de produção que utiliza $n$ inputs $y_{i k}, i=1, \ldots, n$, para produzir $m$ outputs $x_{j k}, j=1, \ldots, m$. Esse modelo maximiza o quociente entre a combinação linear dos outputs e a combinação linear dos inputs, com a restrição de que para qualquer DMU esse quociente não pode ser maior que 1.

Mediante alguns artifícios matemáticos, esse modelo pode ser linearizado, transformando-se em um Problema de Programação Linear apresentado nas equações (1) a (4). O dual deste modelo, dado pelas equações (5) a (9) e conhecido como formulação do envelope, permite determinar a fronteira de eficiência.

$$
\begin{gathered}
\max h_{0}=\sum_{j=1}^{s} u_{j} y_{j 0} \\
\text { sujeito a } \\
\sum_{i=1}^{m} v_{i} x_{i 0}=1 \\
\sum_{j=1}^{s} u_{j} y_{j k}-\sum_{i=1}^{m} v_{i} x_{i k} \leq 0, k=1, \ldots, n \\
u_{j}, v_{i} \geq 0 \quad \forall x, y
\end{gathered}
$$

onde $h_{o}$ é a eficiência da DMU $o$ em análise; $x_{i o}$ e $y_{j o}$ são os inputs e outputs da $\mathrm{DMU}_{o}$; $v_{i}$ e $u_{j}$ são os pesos calculados pelos modelo para inputs e outputs.

$$
\begin{gathered}
\min _{0} \\
\text { sujeito a } \\
h_{0} x_{0} \geq \sum_{j=1}^{n} \lambda_{j} x_{j}
\end{gathered}
$$




$$
\begin{gathered}
y_{0} \leq \sum_{j=1}^{n} \lambda_{j} y_{j} \\
\lambda_{j} \geq 0 \quad \forall j \\
h_{0} \geq 0
\end{gathered}
$$

onde $h_{o}$ é a eficiência da DMU $\mathrm{DM}_{o}$ em análise; $x_{o}$ e $y_{o}$ são os inputs e outputs da $\mathrm{DMU}_{o} ; \mu_{j}$ são as contribuições da DMU $j$ na projeção eficiente da $\mathrm{DMU}_{o} ; x_{j}$ e $y_{j}$ são os inputs e outputs da $\mathrm{DMU}_{j}$.

Outro modelo clássico é o modelo BCC (Banker, Charnes e Cooper, 1984), também chamado de VRS (variable returns to scale) considera situações de eficiência de produção com variação de escala e não assume proporcionalidade entre inputs e outputs. Este modelo não será utilizado neste artigo.

O modelo CCR apresentado considera orientação a inputs, ou seja, minimizar a quantidade de inputs sem alterar a quantidade de outputs. É possível construir modelos com orientação a outputs, nos quais deseja-se maximizar a quantidade de outputs sem alterar os inputs.

\subsection{Método da Fronteira Invertida}

Apesar das vantagens dos modelos DEA, estes geralmente resultam em um grande número de DMUs eficientes. Isso porque, pela formulação matemática, eles tendem a ser benevolentes com as unidades de produção avaliadas (o BCC ainda mais que o CCR) (Soares de Mello et $a l .$, 2005). As principais razões para pouca discriminação entre as DMUs são as seguintes (Angulo Meza e Estellita Lins, 2002):

- O O número de DMUs é pequeno em relação ao número total de variáveis em análise;

- Uma imprecisão ou inadequação no cálculo dos pesos, que pode ser irreal, dando um peso grande para variáveis com menos importância ou dando um peso muito pequeno (ou zero) para variáveis importantes;

- Multiplicidade de soluções ótimas na atribuição de pesos de DMUs fortemente eficientes. 
De acordo com Angulo Meza e Estellita Lins (2002), para solucionar o problema de pouca discriminação de DEA, há dois grandes grupos de métodos: um grupo cujos métodos demandam a incorporação de informações a priori sobre as variáveis (opinião dos tomadores de decisão, por exemplo), que é o caso do método de restrição aos pesos; e outros que não usam tais informações para o cálculo, que é o caso do método da avaliação cruzada.

Posteriormente outros métodos foram propostos, que é o caso do Método da Fronteira Invertida. Baseado no conceito de ranking das DMUs ineficientes, encontrado em Adler et al. (2002), o método da Fronteira Invertida foi proposto inicialmente por Yamada et al. (1994) e posteriormente ilustrado por Entani et al. (2002), os quais denominavam o método de IDEA (“Inverted DEA").

O método da Fronteira Invertida, de acordo com Soares de Mello et al. (2008) e Soares de Mello et al. (2008), é obtido com a inversão entre inputs e outputs. Tal método possui duas interpretações: a primeira é uma análise que conjuga os resultados da fronteira padrão do modelo CCR com a Fronteira Invertida, com isso, a fronteira padrão benevolente destaca só as características positivas e a fronteira invertida destaca as características negativas; já a segunda interpretação é a fronteira formada pelas DMUs com as piores práticas, ou as DMUs eficientes com a ótica invertida. Tal fato pode ser melhor observado em Lins et al. (2005), cujo trabalho aplicou a Fronteira Invertida na interpretação de duas óticas diferentes, vendedor e comprador de imóveis, concluindo que as mesmas variáveis são inputs e outputs diferentes para as duas óticas.

Além disso, o método da Fronteira Invertida faz com que não seja permitida a especialização das DMUs. Esta especialização é permitida pelos modelos CCR e BCC ao permitir que pesos zeros sejam dados às variáveis na análise. Assim, algumas DMUs têm seus pesos não nulos somente em algumas variáveis. A fronteira invertida anula esse efeito destacando também variáveis nas quais as DMUs não tem bom desempenho. Por essa fronteira, as DMUs eficientes são, justamente, as que apresentam os piores desempenhos (fronteira ineficiente) (Soares de Mello et al., 2005). A Figura 1 mostra as duas fronteiras, a clássica e a invertida, para o caso DEA BCC. 


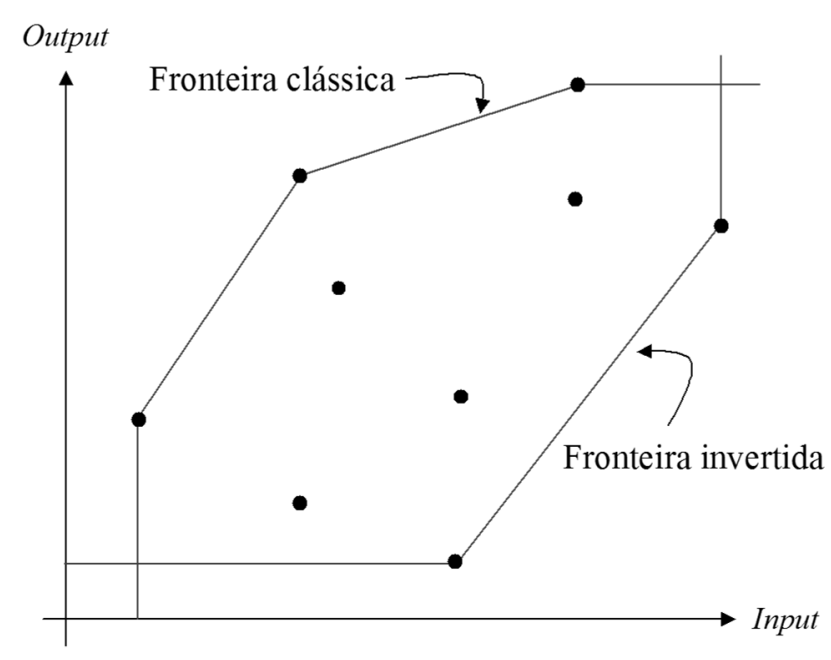

\section{Figura 1 - Fronteiras DEA BCC clássica e invertida ${ }^{1}$}

Com efeito, para os fins deste trabalho, será adotado, conforme conveniência, um índice de eficiência (eficiência composta) que conjuga os aspectos positivos e negativos de cada DMU (média aritmética da eficiência segundo as óticas otimista e pessimista, observada na equação (10) (Soares de Mello et al., 2008).

$$
\text { Eficiência }_{\text {composta }}=\frac{\text { Eficiência }_{\text {padrão }}+\left(1-\text { Eficiência }_{\text {invertida }}\right)}{2}
$$

O índice de eficiência composta (10) representa uma avaliação pela fronteira invertida e que permite uma classificação completa das unidades em estudo. Esse índice é definido pela média aritmética entre a eficiência em relação à fronteira DEA convencional (padrão) e o complemento da eficiência em relação à fronteira invertida, cujo uso é justificado pelo fato da fronteira invertida gerar uma medida de ineficiência.

Neste trabalho será utilizado o modelo CCR com orientação a inputs e análise da fronteira invertida, no processo licitatório de contratação de embarcação de apoio marítimo a fim de garantir a melhoria deste processo pela Petrobras, estabelecendo uma medida para auxiliar no processo de tomada de decisão, baseada em características técnicas e econômicas.

Cabe destacar que apesar de algumas variáveis do modelo não respeitarem a propriedade da proporcionalidade (e.g. custo e velocidade), o modelo CCR é o mais adequado. O modelo BCC

\footnotetext{
${ }^{1}$ Fonte: Soares de Mello et al. (2005)
} 
apresenta alguns problemas teóricos já mencionados na literatura, como eficiências por default (Ali, 1993) e eficiências negativas implícitas tal como descrito por Soares de Mello et al. (2013).

\section{Estudo de Caso}

\subsection{Modelagem}

Como descrito, o objetivo do trabalho é avaliar os processos licitatórios da Petrobrás para contratação de embarcações do tipo PSV 3000 TPB, ou seja, que tenham capacidade de carga de 3000 toneladas através a utilização da técnica de Análise Envoltória de Dados (DEA). A vantagem da utilização de DEA é permitir a inclusão de variáveis econômicas e técnicas envolvidas no processo. Outro ponto importante no método é atribuir pesos às variáveis do modelo sem a interferência do decisor.

Por permitir a incorporação de variáveis não só econômicas, DEA se apresenta como uma forma alternativa de avaliar propostas em um processo licitatório, que atualmente é realizado apenas considerando a variável preço.

Neste trabalho, as DMUs do modelo DEA são as propostas concorrentes de embarcações de apoio marítimo tipo PSV 3000 TPB numa determinada licitação, num total de 14 DMUs, entre as quais se deseja estabelecer um ranking a partir da eficiência de cada proposta, a fim de selecionar a vencedora.

No passo seguinte, são definidas as variáveis que serão utilizadas na modelagem. Para definição destas variáveis, foram analisados os objetivos da contratação das embarcações e as características do problema, descritos a seguir: para garantir o suprimento de cargas para as plataformas, são necessárias que as embarcações tipo PSV possuam área de convés para o transporte de cargas sólidas e tanques para o transporte de líquidos. Tais características devem estar associadas a uma potência e velocidade de navegação adequada ao atendimento as unidades offshore localizadas a quilômetros da costa. Além disso, deve-se minimizar o impacto financeiro através da contratação de embarcações mais econômicas, ou seja, que consumam pouco combustível e que consequentemente emitem menos poluentes atmosféricos, além de pagar taxas diárias de afretamento menores. É importante considerar, ainda, variáveis 
relacionadas ao número de tripulantes necessários para operação e a capacidade de salvaguarda das pessoas abordo da embarcação.

Após análise das características das embarcações entregues pelos licitantes nos anexos técnicos do processo licitatório, foram escolhidas nove variáveis que descrevem o processo de contratação de embarcações de apoio marítimo e mais impactam na atividade de suprimento de plataformas offshore. As variáveis selecionadas consideram aspectos técnicos e econômicos. Das nove variáveis do processo, duas são inputs e sete são outputs. Os inputs são:

- Taxa: valor da taxa diária ofertada para cada embarcação (DMU) (US\$);

- Consumo: consumo de combustível da embarcação (toneladas/dia);

Os outputs são:

- Água: capacidade total de armazenamento de água potável $\left(\mathrm{m}^{3}\right)$;

- Diesel: capacidade total de armazenamento de óleo diesel $\left(\mathrm{m}^{3}\right)$;

- Carga: capacidade total de armazenamento de carga de convés $\left(\mathrm{m}^{2}\right)$;

- Potência: potência máxima contínua de propulsão da embarcação (BHP);

- Velocidade: velocidade máxima a ser atingida durante a navegação da embarcação;

- Tripulantes: número de tripulantes necessários para operação da embarcação;

- Salvatagem: é o conjunto de meios e procedimentos para salvaguardar a vida dos passageiros e tripulantes a bordo da embarcação (número de pessoas atendidas).

As variáveis de input, taxa e consumo, são, respectivamente, as variáveis econômicas do processo de contratação de embarcações, as quais impactam diretamente no dispêndio monetário da Petrobras, já que, apesar da embarcação ser afretada, o combustível é pago pela contratante. Assim, estas variáveis devem ser minimizadas. Os outputs considerados são as "vantagens" obtidas pela Petrobras através do dispêndio com os inputs.

Os outputs água, diesel, carga, potência e velocidade representam variáveis operacionais que impactam no atendimento das unidades marítimas e os outputs tripulantes e salvatagem 
representam variáveis técnicas, econômicas e de segurança das embarcações.

A variável água foi escolhida porque as unidades marítimas necessitam de água potável para consumo, já que a maioria não possui capacidade de tratamento de água salgada para consumo humano. A variável diesel descreve a necessidade do combustível para o funcionamento dos equipamentos de processo das plataformas. A variável carga representa a área de convés da embarcação disponível para o transporte de carga sólida necessária para operação das unidades marítimas. A variável potência foi incluída, pois expressa a capacidade da embarcação conseguir descarregar as cargas transportadas nas plataformas em condições de mar adversas. A variável velocidade representa a rapidez no atendimento das cargas solicitadas pelas unidades marítimas. A variável tripulantes descreve a necessidade mínima de tripulantes para operação da embarcação, e como dois terços dos mesmos devem ser brasileiros, um maior número de tripulantes representa uma maior contribuição para economia brasileira, no que tange o fator de empregabilidade. Num início parece que a este variável é um input, no entanto, seu custo já está considerado na variável Taxa. A variável salvatagem descreve a quantidade máxima de salvamento dos passageiros e tripulantes em caso de emergência nas plataformas ou na própria embarcação.

Foi escolhido o modelo CCR, pois considera retornos constantes de escala, é menos benevolente que o modelo $\mathrm{BCC}$ e, como as embarcações possuem tecnologias semelhantes ou operam nas mesmas condições, este modelo é o mais adequado. $\mathrm{O}$ modelo será orientado à input, devido ao interesse da redução dos custos da embarcação tipo PSV 3000 TPB, mantendo as características técnicas constantes.

\subsection{Análise e Discussão dos Resultados}

A Tabela 1 apresenta os dados para 14 embarcações utilizados na modelagem. Estes dados foram obtidos de um processo licitatório realizado em 2009 para embarcações do tipo PSV 3000 TPB, cujo resultado já se tornou público. 
Tabela 1 - Dados de embarcações tipo PSV 3000²

\begin{tabular}{|c|c|c|c|c|c|c|c|c|c|c|}
\hline \multirow{2}{*}{$\begin{array}{c}\text { Embarcação } \\
(\text { DMU) }\end{array}$} & \multicolumn{2}{|c|}{ INPUTS } & \multicolumn{7}{|c|}{ OUTPUTS } \\
\cline { 2 - 11 } & Taxa & Consumo & Água & Diesel & Carga & Potência & Vel. & Trip. & Salv. \\
\hline ACF & 22200 & 22,9 & 1500 & 800 & 540 & 4600 & 13 & 16 & 30 \\
\hline DT & 19980 & 22 & 1450 & 800 & 585 & 5450 & 14 & 12 & 25 \\
\hline HP & 21900 & 20,6 & 1450 & 800 & 611 & 6038 & 11,5 & 12 & 25 \\
\hline HW & 18670 & 14,5 & 1450 & 800 & 570 & 5300 & 11 & 13 & 80 \\
\hline HB & 22400 & 15 & 1500 & 800 & 730 & 4000 & 12 & 13 & 16 \\
\hline HGE & 21900 & 15 & 1500 & 800 & 730 & 4000 & 12 & 13 & 16 \\
\hline HGR & 21900 & 15 & 1500 & 800 & 730 & 4000 & 12 & 13 & 16 \\
\hline HN & 21400 & 26 & 1500 & 800 & 615 & 7800 & 12 & 13 & 16 \\
\hline KC & 21300 & 27 & 1500 & 800 & 576 & 5500 & 11 & 12 & 20 \\
\hline LT & 18670 & 22 & 1450 & 800 & 585 & 5460 & 14 & 12 & 25 \\
\hline NT & 21900 & 22,5 & 1500 & 800 & 564 & 5400 & 12 & 12 & 48 \\
\hline NV & 21900 & 22,5 & 1500 & 800 & 564 & 5400 & 12 & 12 & 25 \\
\hline SC & 21900 & 16,5 & 1500 & 1000 & 625 & 6470 & 13 & 12 & 20 \\
\hline SS & 20500 & 23 & 1500 & 800 & 572 & 5460 & 13 & 15 & 20 \\
\hline
\end{tabular}

A Tabela 2 mostra a ordenação das PSVs baseada na forma atual de contratação, descrita como "menor preço", ou seja, se as embarcações têm os requisitos técnicos mínimos para atendimento da demanda, as que tiverem os menores preços vencem a licitação. Assim, as primeiras colocadas do processo licitatório foram às embarcações HW e LT, por terem oferecido a menor Taxa Diária.

Pela Tabela 2, observa-se um empate entre a primeira e segunda colocada, o que dificulta a determinação da proposta vencedora, já que as duas embarcações pertencem a empresas diferentes. Nesse processo licitatório específico, a Comissão de Licitação não teve maiores problemas porque a demanda a ser atendida era para mais de uma embarcação.

\footnotetext{
${ }^{2}$ Fonte: os autores.
} 
Tabela 2 - Ordenação das propostas de acordo com a taxa diária ofertada ${ }^{3}$

\begin{tabular}{|c|c|}
\hline Embarcações PSVs & Taxa Diária (USD) \\
\hline HW & 18670 \\
\hline LT & 18670 \\
\hline DT & 19980 \\
\hline SS & 20500 \\
\hline KC & 21300 \\
\hline HN & 21400 \\
\hline HP & 21900 \\
\hline HGE & 21900 \\
\hline HGR & 21900 \\
\hline NT & 21900 \\
\hline NV & 21900 \\
\hline SC & 21900 \\
\hline ACF & 22200 \\
\hline HB & 22400 \\
\hline
\end{tabular}

Utilizando os dados apresentados na Tabela 1, foi utilizado o software SIAD (Angulo Meza et $a l ., 2005)$ para determinação das eficiências das DMUs. O software fornece também o valor das eficiências pela técnica da fronteira invertida e o valor das eficiências compostas segundo a equação (V). O valor das eficiências compostas das DMUs estão apresentados na Tabela 3, que mostra também o valor da taxa diária (US\$) para as embarcações. A ordenação das embarcações nesta tabela é feita segundo a eficiência composta, expressa em porcentagem.

Na Tabela 3, observa-se que a embarcação melhor posicionada foi a HW, a qual apresentou a maior eficiência composta. Esta embarcação foi a vencedora do processo licitatório por possuir a menor taxa diária. Pelo método aqui apresentado, esta embarcação possui ainda, além do menor preço, as melhores características técnicas e econômicas, a qual seria ideal para contratos de longo prazo, devido ao menor risco de se ter problemas operacionais, o que geralmente não ocorre quando a escolha se dá apenas pelo menor preço.

\footnotetext{
${ }^{3}$ Fonte: os autores
} 
Tabela 3 - Eficiência composta das embarcações ${ }^{4}$

\begin{tabular}{|c|c|c|}
\hline $\begin{array}{c}\text { DMUs } \\
\text { (Embarcações PSVs) }\end{array}$ & $\begin{array}{c}\text { Taxa Diária } \\
\text { (USD) }\end{array}$ & $\begin{array}{c}\text { Eficiência } \\
\text { Composta }(\%)\end{array}$ \\
\hline HW & 18670 & $100.00 \%$ \\
\hline LT & 18670 & $99.94 \%$ \\
\hline SS & 20500 & $93.09 \%$ \\
\hline DT & 19980 & $92.65 \%$ \\
\hline HGE & 21900 & $91.25 \%$ \\
\hline HGR & 21900 & $91.25 \%$ \\
\hline HB & 22400 & $90.20 \%$ \\
\hline HN & 21400 & $90.20 \%$ \\
\hline SC & 21900 & $90.20 \%$ \\
\hline ACF & 22200 & $89.98 \%$ \\
\hline HP & 21900 & $82.84 \%$ \\
\hline NT & 21300 & $81.79 \%$ \\
\hline NV & 21900 & $79.55 \%$ \\
\hline & 21900 & $79.55 \%$ \\
\hline
\end{tabular}

Cabe ressaltar ainda que, através da metodologia proposta, a embarcação HW é mais eficiente que a embarcação LT, ou seja, a embarcação HW é tecnicamente e socialmente superior, já que existe um empate econômico entre as duas propostas, resolvendo assim o possível problema de seleção apresentado na Tabela 2.

A Tabela 4 apresenta os rankings obtidos pelo processo licitatório tradicional e o ranking do modelo DEA proposto. Pode-se notar que as duas primeiras posições permanecem inalteradas nos dois rankings, entretanto, no ranking DEA, há um desempate entre elas. A partir da terceira posição, existe uma alteração entre eles.

Comparando este resultado com o obtido em Maciel e Angulo Meza (2012) observa-se que não se alteram as duas primeiras colocações, principalmente por apresentarem altos valores de salvatagem. A principal mudança verifica-se na embarcação SS que anteriormente estava no $8^{\circ}$ lugar, isso é devido à quantidade de tripulantes.

\footnotetext{
${ }^{4}$ Fonte: os autores
} 
Tabela 4 - Rankings modelo tradicional e modelo DEA ${ }^{5}$

\begin{tabular}{|c|c|c|c|}
\hline $\begin{array}{c}\text { Ranking } \\
\text { Tradicional }\end{array}$ & $\begin{array}{c}\text { Método } \\
\text { Tradicional }\end{array}$ & $\begin{array}{c}\text { Ranking } \\
\text { DEA }\end{array}$ & Método DEA \\
\hline 1 & HW & 1 & HW \\
\hline 1 & LT & 2 & LT \\
\hline 3 & DT & 3 & SS \\
\hline 4 & SS & 4 & DT \\
\hline 5 & KC & 5 & HGE \\
\hline 6 & HN & 6 & HGR \\
\hline 7 & HP & 7 & HB \\
\hline 8 & HGE & 8 & HN \\
\hline 9 & HGR & 9 & SC \\
\hline 10 & NT & 10 & ACF \\
\hline 11 & NV & 11 & HP \\
\hline 12 & SC & 12 & KC \\
\hline 13 & ACF & 13 & NT \\
\hline 14 & HB & 14 & NV \\
\hline
\end{tabular}

\section{Conclusão}

Este trabalho avaliou o processo licitatório da Petrobrás para contratação de embarcações do tipo PSV 3000 TPB, ou seja, que tenham capacidade de carga de 3000 toneladas através a utilização da técnica de Análise Envoltória de Dados (DEA). A utilização de DEA se justificou por sua capacidade de considerar variáveis econômicas e técnicas envolvidas no processo, além de não ter sido necessária a intervenção do decisor na atribuição dos pesos destas variáveis. Além disso, a inclusão das variáveis Tripulantes e Salvatagem permitiu maior distinção entre as propostas.

O método tradicional de licitações pode favorecer a contratação de recursos que possivelmente terão problemas operacionais no futuro, pela escolha da proposta vencedora ser baseada apenas no custo, sendo mais apropriado em contratações de curto prazo. Em contratações de médio e longo prazo, a escolha de uma proposta vencedora pelo menor custo pode ser uma grande armadilha para a organização, pois podem surgir outros custos, como por exemplo, de

\footnotetext{
${ }^{5}$ Fonte: os autores
} 
manutenção, que elevaria muito o custo inicial.

Neste sentido a metodologia DEA proposta para análise de processos licitatórios neste trabalho buscou agregar valores técnicos e operacionais na contratação de recursos. A utilização de DEA para cálculo da eficiência das propostas concorrentes e ranqueamento destas propostas permitiu a incorporação de variáveis técnicas ao processo. Além disso, o uso do método da fronteira invertida aumentou a discriminação das propostas concorrentes sem necessitar da interferência do decisor, garantindo assim a imparcialidade do processo licitatório. Tal método permitiu ainda selecionar a proposta com bom desempenho nas suas melhores características e que, também, não possui desempenho ruim nas suas piores características.

Os resultados mostram também que a utilização do modelo proposto, a alternativa de "melhor preço" vence, ou seja, a proposta que possui um melhor conjunto de variáveis técnicas e econômicas vence o processo licitatório, tendo como base a análise conjunta dos critérios técnicos e econômicos. Sendo assim, é possível observar que tal método favorece a contratação da melhor embarcação para atendimento as atividades operacionais.

Para trabalhos futuros, propõe-se a utilização de outros modelos de DEA para determinar melhoras que possam ser implementadas nas propostas de forma a atingirem o primeiro lugar no ranking. 


\section{Referências}

Adler, N., Friedman, L. e Sinuany-Stern, Z. (2002). Review of ranking methods in the data envelopment analysis context. European Journal of Operational Research, vol. 140, pp. 249-265.

Aizemberg, L., Roboredo, M. C., Ramos, T. G., Angulo-Meza, L. e Soares de Mello, J. C. C. B. (2011). Measuring the NBA teams' cross-efficiency by DEA. 3rd IMA - International Conference on Mathematics in Sport, Manchester.

Ali, A. I. (1993). Streamlined computation for data envelopment analysis. European Journal of Operational Research, vol. 64, pp. 61-67.

Angulo Meza, L. e Estellita Lins, M. P. (2002). Review of methods for increasing discrimination in data envelopment analysis. Annals of Operations Research, vol. 116, pp. 225-242.

Angulo Meza, L., Biondi Neto, L., Soares de Mello, J. C. C. B. e Gomes, E. G. (2005). ISYDS - Integrated System for Decision Support (SIAD Sistema Integrado de Apoio a Decisão): A Software Package for Data Envelopment Analysis Model. Pesquisa Operacional, vol. 25, n. 3, pp. 493-503.

Banker, R.D., Charnes, A., e Cooper, W. W. (1984). Some models for estimating technical scale inefficiencies in data envelopment analysis. Management Science, vol. 30, n. 9, pp. 10781092.

Bougnol, M. L., Dulá, J. H., Estellita Lins, M. P. e Moreira da Silva, A. C. (2010). Enhancing standard performance practices with DEA. Omega, vol. 38, n. 1-2, pp. 33-45.

Caillaux, M. A., Sant'anna, A. P., Angulo Meza, L. e Soares de Mello, J. C. C. B. (2011). Container Logistics in Mercosur: Choice of a Transhipment Port Using Ordinal Copeland Method, Data Envelopment Analysis and Probabilistic Composition. Maritime Economics and Logistics, vol. 13, n. 4, pp. 355-370.

Charnes, A., Cooper, W.W. e Rhodes, E. (1978). Measuring the efficiency of decision-making units. European Journal of Operational Research, vol. 2, pp. 429-444.

Cooper, W. W., Seiford, L. e Tone, K. (2000). Data envelopment analysis: A comprehensive text with models, applications, references and DEA-solver software. Boston: Kluwer.

Cortez, L. C. S., Oliveira, L. R., Martins, E. F., Jesus, I. R. D. e Soares de Mello, J. C. C. B.. (2013). Análise de eficiência na gestão de portos públicos brasileiros em relação ao papel das autoridades portuárias. Journal of Transport Literature, vol. 7, pp. 78-96.

Dai, X. e Kuosmanen, T. (2014). Best-practice benchmarking using clustering methods: Application to energy regulation. Omega, vol. 42, n. 1, pp.179-188.

Doyle, J. R. e Green, R. H. (1994). Efficiency and cross-efficiency in DEA derivations, meanings and uses. Journal of the Operational Research Society, vol. 45, pp. 567-578.

El-Mashaleh, M. S. (2010). Decision to bid or not to bid: A data envelopment analysis approach. Canadian Journal of Civil Engineering, vol. 37, n. 1, pp. 37-44.

El-Mashaleh, M. S. (2013). Empirical framework for making the Bid/No-Bid decision. Journal of Management in Engineering, vol. 29, n. 3, pp. 200-205. 
Entani, T., Maeda, Y. e Tanaka, H. (2002). Dual Models of Interval DEA and its extensions to interval data. European Journal of Operational Research, vol. 136, pp. 32-45.

Falagario, M., Sciancalepore, F., Costantino, N. e Pietroforte, R. (2012). Using a DEA-cross efficiency approach in public procurement tenders. European Journal of Operational Research, vol. 218, n. 2, pp. 523-529.

Fernandes, J. E. M., Gomes, L. F. A. M., Soares de Mello, J. C. C. B. e Gomes Júnior, S. F. (2013). Seleção de uma aeronave para atividades de charter regional com utilização do método multicritério de borda modificado com utilização de mediana. Journal of Transport Literature, vol. 7, pp. 171-191.

Ferrari, C. e Basta, M. (2009). Port concession fees based on the price-cap regulation: A DEA approach. Maritime Economics and Logistics, vol. 11, n. 1, pp. 121-135.

Gabriele, P. D., Brandão, L. C., Treinta, F. T. M., Soares de Mello, J. C. C. B. e Carvalhal, R. (2013). Comparação internacional da eficiência ambiental dos modos de transporte rodoviário e ferroviário. Journal of Transport Literature, vol. 7, pp. 212-229.

Garcia-Alonso, L. e Martin-Bofarull, M. (2007). Impact of port investment on efficiency and capacity to attract traffic in Spain: Bilbao versus Valencia. Maritime Economics and Logistics, vol. 9, n. 3, pp. 254-267.

Gomes, E. G., Soares de Mello, J. C. C. B., Souza, G. D. S., Angulo Meza, L. e Mangabeira, J. A. D. C. (2009). Efficiency and sustainability assessment for a group of farmers in the Brazilian Amazon. Annals of Operations Research, vol. 169, n. 1, pp. 167-181.

Haralambides, H., Hussain, M., Barros, C.P. e Peypoch, N. (2010). A new approach in benchmarking seaport efficiency and technological change. International Journal of Transport Economics, vol. 37, n. 1, pp. 77-96.

Leenders, M. R., Johnson, P. F., Flynn, A. E. e Fearon, H. E. (2006). Purchasing and Supply Management. New York: McGraw Hill-Irwin.

Li, L., Wu, C. L., Li, Y. Q., Xia, H. e Wang, Y. (2010). System analysis for contractor prequalification based on $P-D E A$ method.

Li, X. G., Yang, J. e Liu, X. J. (2013). Analysis of Beijing's environmental efficiency and related factors using a DEA model that considers undesirable outputs. Mathematical and Computer Modelling, vol. 58, n. 5-6, pp. 956-960.

Li, X.-B. e Reeves, G. R. (1999). A multiple criteria approach to data envelopment analysis. European Journal of Operational Research, vol. 115, n. 3, pp. 507-517.

Lins, M. P. E., De Lyra Novaes, L. F. e Legey, L. F. L. (2005). Real estate appraisal: A double perspective data envelopment analysis approach. Annals of Operations Research, vol. 138. n. 1, pp. 79-96.

Lorentziadis, P. L. (2010). Post-objective determination of weights of the evaluation factors in public procurement tenders. European Journal of Operational Research, vol. 200, n.1, pp. 261-267.

Maciel, G. S. e Angulo Meza, L. (2012). Aplicação de DEA em Processos Licitatórios e Análise dos Processos Estratégicos de Inovação Aplicados ao Estudo de Caso de Embarcações Offshore da Petrobras. Relatório de Pesquisa em Engenharia de Produção (UFF), vol. 12 , pp. 53-68. 
Managi, S. (2007). Maritime shipping industry and productivity in Japan. Maritime Economics and Logistics, vol. 9, n. 4, pp. 291-301.

Menegaki, A. N. (2013). Growth and renewable energy in Europe: Benchmarking with data envelopment analysis. Renewable Energy, vol. 60, pp. 363-369.

Narasimhan, R., Talluri, S. e Mendez, D. (2001). Supplier evaluation and rationalization via data envelopment analysis: An empirical approach. Journal of Supply Chain Management, vol. 37, n. 3, pp. 28-37.

Ohno, T. e Harada, Y. (2006). Comparison of tendering and contracting systems for Public works between Japan, the United States and EU countries. Government Auditing Review - Board of Audit Japan, vol. 13, pp. 49-71.

Pereira, M. F. (1995). Mensuramento de Eficiência Multidimensional utilizando Análise de Envelopamento de Dados: Revisão da Teoria e Aplicações. Dissertação (Mestrado em Engenharia de Produção), Universidade Federal de Santa Catarina, Florianópolis.

Saranga, H. (2007). Multiple objective data envelopment analysis as applied to the Indian Pharmaceutical Industry. Journal of the Operational Research Society, vol. 58, n. 11, pp. 1480-1493.

Sexton, T. R., Silkman, R.H. e Logan, A.J. (1986). Data Envelopment Analysis: Critique and extensions. In H. Silkman (Ed.), Measuring efficiency: An assessment of data envelopment analysis. San Francisco: Jossey-Bass Editor, pp. 73-105.

Shuang, Q., Yuan, Y. e Shuang, K. (2011) Bidding evaluation of construction engineering using extend data envelopment analysis method. Advanced Materials Research, vol. 243-249, pp. 6369-6372.

Soares de Mello, J. C. C. B., Angulo-Meza, L., Gomes, E. G. e Biondi Neto, L. (2005). Curso de Análise de Envoltória de Dados. XXXVII SBPO - Simpósio Brasileiro de Pesquisa Operacional, Gramado.

Soares de Mello, J. C. C. B., Angulo-Meza, L., Gomes, E. G., Fernandes, A. J. S. e Biondi Neto, L. (2008). Estudo não paramétrico da relação entre consumo de energia, renda e temperatura. IEEE Latin America Transactions, vol. 6, n. 2, pp. 153-161.

Soares de Mello, J. C. C. B., Angulo Meza, L., Silveira, J. Q. e Gomes, E. G.. (2013). About negative efficiencies in Cross Evaluation BCC input oriented models. European Journal of Operacional Research, vol. 229, pp. 732-737.

Soares de Mello, J. C. C. B., Gomes, E. G., Angulo-Meza, L. e Leta, F. R. (2008). DEA Advanced Models for Geometric Evaluation of used Lathes. WSEAS Transactions on Systems, vol. 7, n. 5, pp. 500-520.

Yamada, Y., Matui, T. e Sugiyama, M. (1994). New analysis of efficiency based on DEA. Journal of the Operations Research Society of Japan, vol. 37, n. 2, pp. 158-167.

Zhu, H., Wu, J. e Zhao, Y. (2007). A quadratic programming approach to determine the uncertain weights in multiple criteria decision-making problems. 